\title{
Pancreatitis autoinmune asociada a fibrosis retroperitoneal: evolución tras dos años de seguimiento
}

\author{
M. Romero, M. J. Pérez-Grueso, A. Repiso, G. de la Cruz, A. García Vela, R. Martín Escobedo, \\ C. González de Frutos y J. M. Carrobles
}

Servicio de Aparato Digestivo. Hospital Virgen de la Salud. Toledo

\begin{abstract}
RESUMEN
Introducción: la pancreatitis autoinmune es un tipo de pancreatitis crónica caracterizado por un infiltrado linfoplasmocitario y una elevación de IgG e IgG4, que se ha descrito asociada a diversas manifestaciones extrapancreáticas y enfermedades autoinmunes, lo cual apoya la teoría de un mecanismo autoinmune fisiopatólogico de base.

Caso clínico: presentamos el caso de un varón que debutó simultáneamente con una pancreatitis autoinmune asociada a fibrosis retroperitoneal y lesión de la vía biliar extrapancreática, con respuesta total tras tratamiento con corticoides durante 4 meses y ausencia de recurrencia tras 24 meses de seguimiento.

Discusión: la pancreatitis autoinmune es un tipo de pancreatitis crónica que probablemente forme parte de un proceso sistémico autoinmune, cuyas manifestaciones extrapancreáticas más frecuentes son la fibrosis retroperitoneal y las lesiones de la vía biliar extrapancreática. Su correcto diagnóstico e inicio precoz del tratamiento puede favorecer la resolución completa de las lesiones, principalmente en los casos de bajo grado de actividad, con menor probabilidad de recurrencia.
\end{abstract}

Palabras clave: Pancreattis autoinmune. Lesiones extrapancreáticas. Fibrosis retroperitoneal.

\begin{abstract}
Introduction: autoimmune pancreatitis is a kind of chronic pancreatitis characterized by the presence of lymphoplasmacytic infiltration and severely elevated serum IgG and IgG4, which has been associated to many extrapancreatic lesions and other autoimmune disorders, leading to the theory of an autoimmune mechanism involved in the pathogenesis of this disease.

Case report: we report the case of a man who simultaneously presented with autoimmune pancreatitis associated with retroperitonal fibrosis, and a lesion of the extrapancreatic bile duct, with total response to corticosteroid treatment for 4 moths and absence of recurrence after 24 months of follow-up.

Discussion: autoimmune pancreatitis is a kind of chronic pancreatitis that is probably a part of a systemic autoinmune disease, with retroperitoneal fibrosis and extrapancreatic bile duct lesion being the most commonly associated extrapancreatic lesions. A correct diagnosis and early treatment of this disease may aid in the total resolution of lesions, especially in cases with a low activity grade.
\end{abstract}

Key words: Autoimmune pancreatitis. Extrapancreatic lesion. Retroperitoneal fibrosis.

Romero M, Pérez-Grueso MJ, Repiso A, de la Cruz G, García Vela A, Martín Escobedo R, González de Frutos C, Carrobles JM. Pancreatitis autoinmune asociada a fibrosis retroperitoneal: evolución tras dos años de seguimiento. Rev Esp Enferm Dig 2008; 100: 648-651.

\section{INTRODUCCIÓN}

La pancreatitis autoinmune (PAI) es una forma de pancreatitis crónica, caracterizada por la existencia de un infiltrado linfoplasmocitario pancreático y una elevada concentración de IgG-4 sérica. Se ha descrito su asociación con distintas enfermedades autoinmunes y una variedad de le-

Recibido: $15-04-08$

Aceptado: 29-04-08.

Correspondencia: María José Pérez-Grueso Macías. Servicio de Aparato Digestivo. Hospital Virgen de la Salud. Avda. Barber, 30. 45004 Toledo. e-mail: mjperezgrueso@hotmail.com siones extrapancreáticas (1-3). Por ello algunos autores han postulado la existencia de una enfermedad sistémica fibroesclerosante asociada a IgG-4, en la cual la PAI sería una manifestación más $(1,3,4)$. Presentamos el caso de un varón diagnosticado de PAI que asocia fibrosis retroperitoneal, con una respuesta total al tratamiento con esteroides y sin recurrencia tras 2 años de seguimiento.

\section{CASO CLÍNICO}

Varón de 43 años originario de Marruecos, que ingresó en nuestro servicio por cuadro de 4 días de evolución de 
ictericia mucocutánea indolora, coluria y prurito generalizado. Como únicos antecedentes de interés destacaban una tuberculosis tratada, hipercolesterolemia en tratamiento dietético y tabaquismo leve. La analítica mostró los siguientes datos: bilirrubina $19,44 \mathrm{mg} / \mathrm{dl}$, GOT 110 UI/ml, GPT 371 UI/ml, GGT 632 UI/ml, FA 787 UI/ml, LDH $394 \mathrm{UI} / \mathrm{ml}$. Amilasa y lipasa fueron normales en todo momento. Destacaba también una hipergammaglobulinemia (gammaglobulina 1,56\%), con IgG 1840 $\mathrm{UI} / \mathrm{ml}$ (751-1.560 UI/ml). El marcador CA 19-9 era de 82 $\mathrm{UI} / \mathrm{ml}$. Los autoanticuerpos (ANA, AMA, AMA-2, ANCA, LKM1, SMA, SLA y F-actina) y serologías para virus y bacterias (VHA, VHB, VHC, HIV, CMV, VEB, VVZ, VHS, virus de la parotidis, parvovirus B19, Brucella, Borrelia, Coxiella, Leishmania, Rickettsias y Toxoplasma) fueron negativos. La ecografía abdominal mostró dilatación de la vía biliar intra- y extrahepática y la ecoendoscopia (USE) un páncreas aumentado de tamaño con una lesión focal de $3 \mathrm{~cm}$ a nivel de cabeza y leve dilatación posterior del conducto pancreático. La TAC toracoabdominal objetivó además un páncreas aumentado globalmente de tamaño, hipodenso, con un halo peripancreático hipodenso, con borde liso, sin afectación de la grasa peripancreática, y, a nivel retroperitoneal, en localización periaórtica con extensión a territorio iliaco bilateral, un tejido denso rodeando dichas estructuras vasculares como un estuche, siendo todo ello compatible con pancreatitis autoinmune con fibrosis retroperitoneal (Fig. 1). Ante la persistencia de prurito y la colestasis manifiesta se realizó un drenaje biliar percutáneo mostrando la colangiografía una lesión estenosante en colédoco distal sugerente de neoplasia pancreática. Ante dicha posibilidad como primer diagnóstico diferencial, se realizó una punción-aspiración con aguja fina guiada por USE con citología negativa para células malignas y tinción Zielh negativa. Dada la ausencia de confirmación de neoplasia, y con la sospecha de pancreatitis autoinmune, se realizó una colangiopancreatografía retrógrada endoscópica (CPRE) observando dilatación de la vía biliar principal con una estenosis regular en su porción distal, de unos 3$4 \mathrm{~cm}$, de aspecto benigno, sobre la que se colocó una endoprótesis plástica. Se inició tratamiento con corticoides (metilprednisolona a dosis de $32 \mathrm{mg} /$ día) y se retiró el drenaje percutáneo. Con el diagnóstico de probable PAI el paciente fue dado de alta y seguido de forma ambulatoria comprobando normalización de todos los parámetros analíticos y la resolución en la TAC abdominal de control, al mes y a los 4 meses, de las lesiones descritas inicialmente, con normalización del tamaño y aspecto pancreático y desaparición de la fibrosis retroperitoneal (Fig. 2). La dosis de corticoides fue disminuyéndose progresivamente hasta su retirada completa al $4^{\circ}$ mes, retirándose también la endoprótesis biliar. Hasta la fecha actual, tras 24 meses de seguimiento, el paciente ha permanecido asintomático sin presentar recurrencia de la pancreatitis autoinmune ni de la fibrosis retroperitoneal.
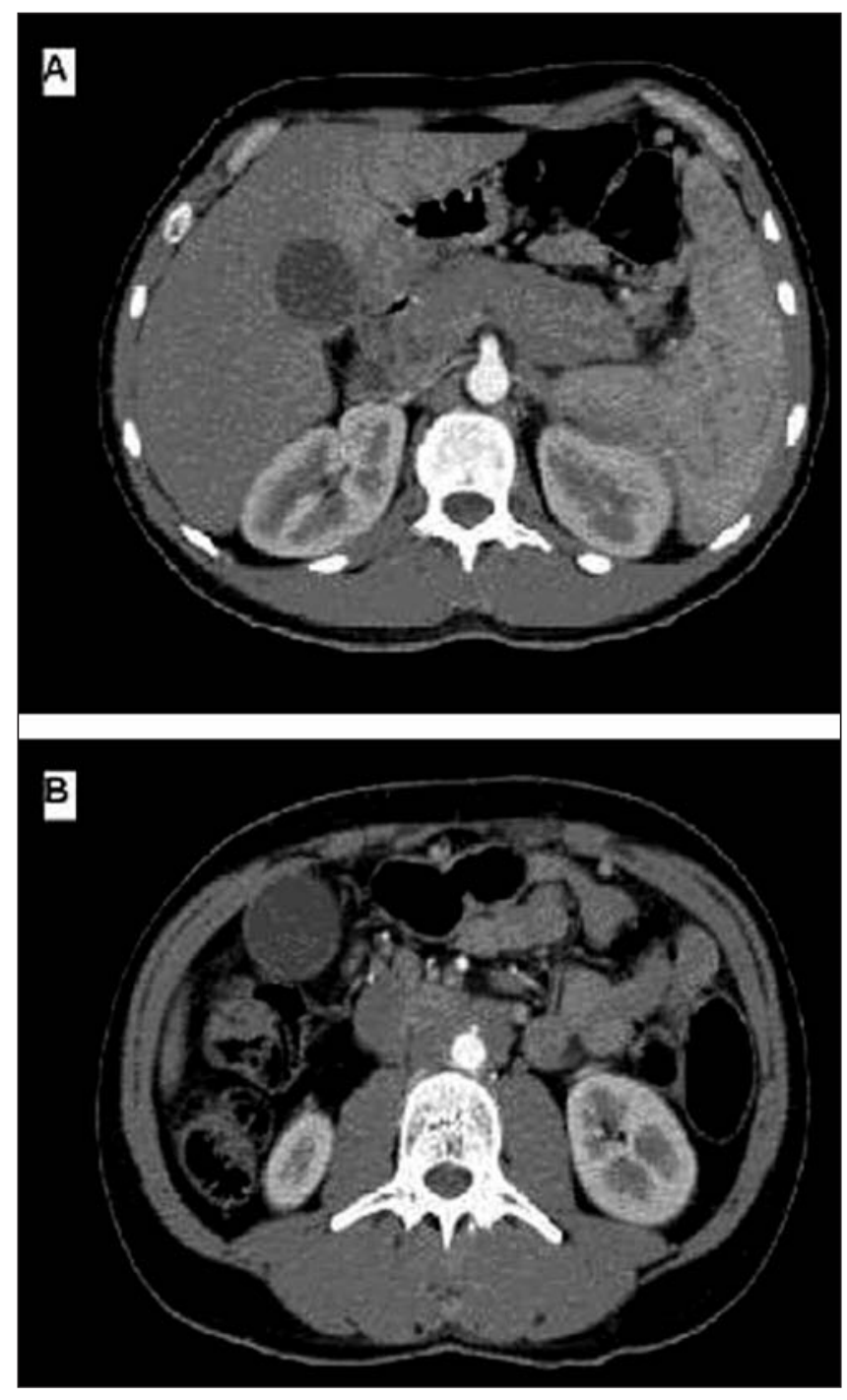

Fig. 1. TAC abdominal. A: Páncreas engrosado de tamaño. B: Fibrosis retroperitoneal periaórtica.

\section{DISCUSIÓN}

La pancreatitis autoinmune (PAI) fue descrita por primera vez por Sarles y cols. en 1961 (5). Es una forma de pancreatitis crónica caracterizada por un infiltrado linfoplasmocitario en el tejido pancreático con fibrosis extensa y una elevada concentración sérica de $\operatorname{IgG}$ e IgG4 $(1,2)$. Las distintas series sugieren una prevalencia del 4,6-6\% de todas las pancreatitis crónicas (3) y afecta más frecuentemente a varones de edad media como es nuestro caso. La presentación clínica más frecuente es la ictericia obstructiva indolora. El hallazgo analítico más característico, objetivado en nuestro paciente, es la hipergammaglobulinemia y el aumento de $\mathrm{IgG}$, presentes en el $60 \mathrm{y}$ $70 \%$ de los casos respectivamente, siendo más específica aún la IgG4 (en el 90\%) (2) que en nuestro caso no fue posible determinar. La IgG4 es un marcador diagnóstico 

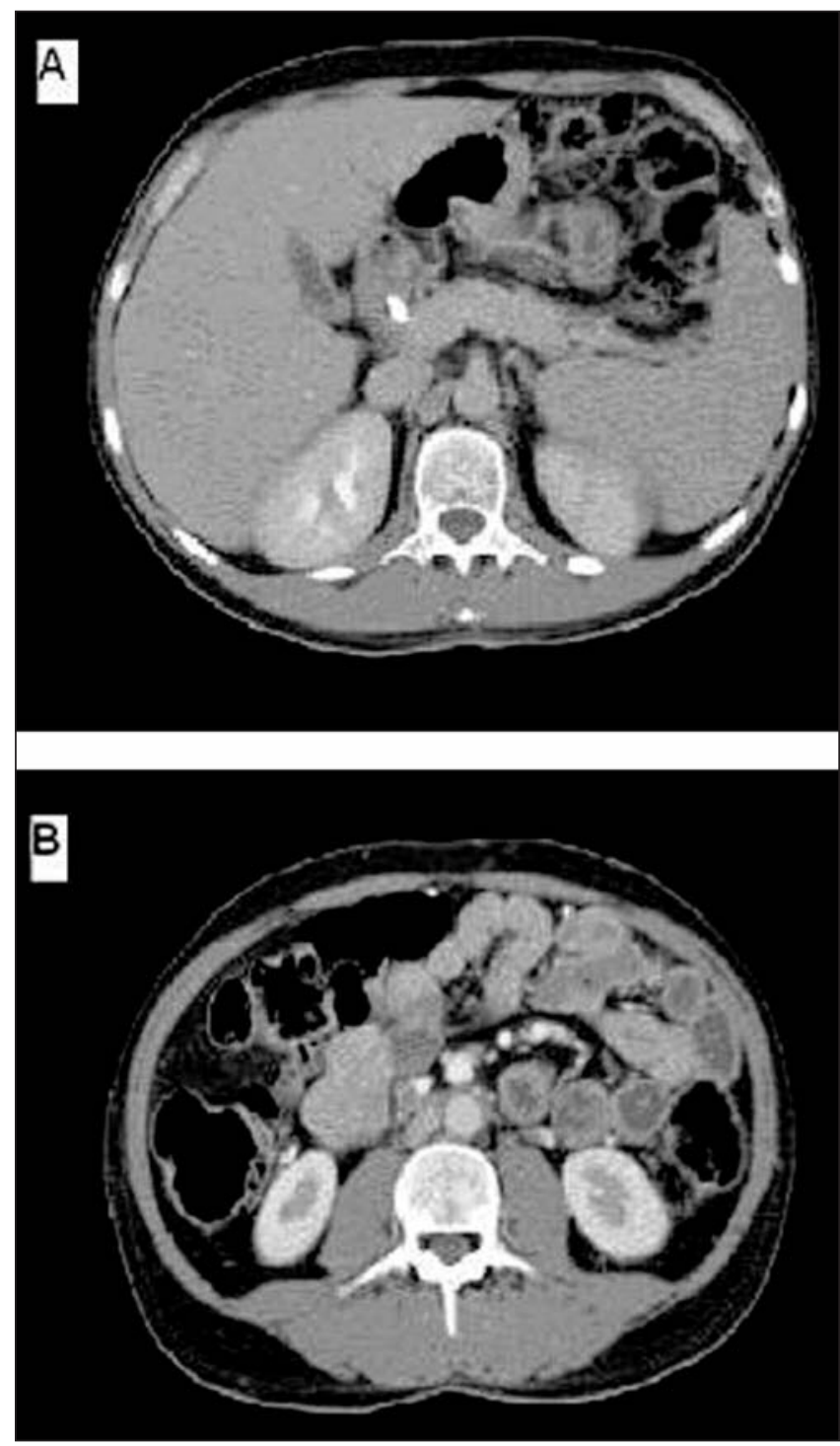

Fig. 2. TAC abdominal tras tratamiento. A: páncreas de tamaño y estructura normal. B: Desaparición de la fibrosis retroperitoneal periaórtica.

con una sensibilidad y especificidad del 90 y $98 \%$ respectivamente para diferenciar la PAI del cáncer de páncreas así como de otros procesos pancreáticos $(2,4)$. El CA 199 suele estar elevado en el 53\% (2) y suele normalizarse tras el tratamiento. La ecografía y la TAC suelen mostrar un aumento difuso del páncreas (saussage-like) y la CPRE una estenosis irregular del conducto pancreático principal (2). La Sociedad Japonesa del Páncreas propuso en 2002 unos criterios para el diagnóstico de PAI, basados en hallazgos de laboratorio, de imagen e histopatólogicos (Tabla I), pero a medida que se han descrito nuevos casos, diversos grupos han propuesto nuevos criterios diagnósticos, entre los que se incluyen la asociación con otras enfermedades autoinmunes y la respuesta al tratamiento con corticoides (6). En los últimos años se ha descrito cada vez con mayor frecuencia una variedad de lesio-
Tabla I. Criterios diagnósticos de pancreatitis autoinmune (Japan Pancreas Society)

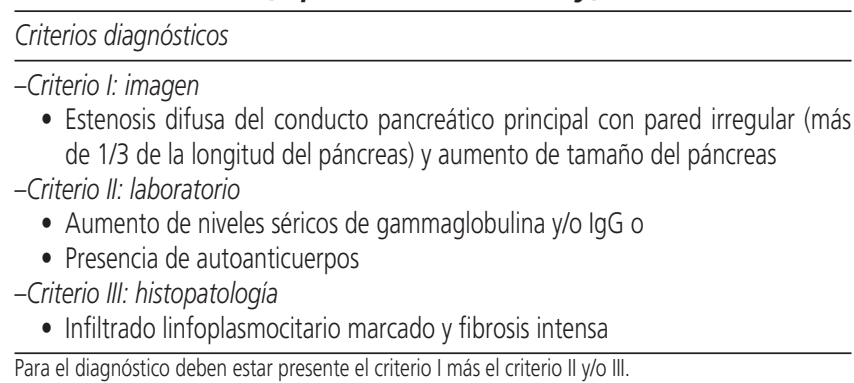

nes extrapancreáticas y algunas enfermedades autoinmunes asociadas, como el síndrome de Sjögren, colangitis esclerosante, fibrosis retroperitoneal, hipotiroidismo, etc. La prevalencia de las lesiones extrapancreáticas tampoco está bien determinada y varía en las distintas series entre el 20-50\% (6). Hasta el año 2006 sólo se habían publicado 9 casos de PAI y fibrosis retroperitoneal $(7,8)$, aunque el mayor conocimiento de esta entidad en los últimos años ha hecho que cada vez se diagnostiquen más casos. Según las series más recientes publicadas, las manifestaciones extrapancreáticas más frecuentes son las lesiones de la vía biliar extrapancreática $(73,9 \%)$ y la fibrosis retroperitoneal $(12,5-66 \%)(1,3)$. Esta última suele presentarse como una lesión de tejidos blandos a nivel de la aorta infrarrenal que puede englobar estructuras adyacentes (3). Las lesiones de la vía biliar extrapancreática más frecuentes son la dilatación difusa de las vías biliares y la estenosis única del colédoco distal (9), que es el tipo de lesión objetivada en nuestro caso. Su hallazgo plantea el diagnóstico diferencial con el cáncer de cabeza de páncreas, por lo que en muchas ocasiones, como en nuestro paciente, obliga a la realización de una PAAF o microbiopsia.

Esta asociación a distintas enfermedades autoinmunes y lesiones extrapancreáticas apoya la teoría de un mecanismo autoinmune como responsable de su fisiopatogenia $(1,2,4,9-11)$ y diversos autores sugieren que realmente se trate de una enfermedad inflamatoria sistémica esclerosante asociada a IgG4 y que la PAI sea una manifestación más de la misma $(1,6-9,11,12)$. Recientemente algunos estudios han señalado que la gammaglobulina y la IgG4 están significativamente más elevadas en la PAI con mayor número lesiones extrapancreáticas (más de 3) que cuando estas no existen (9), y parecen ser un reflejo del grado de actividad del proceso. En nuestro caso el diagnóstico, tanto por las pruebas de imagen como por la asociación con fibrosis retroperitoneal y afectación de la vía biliar extrapancreática y por la buena respuesta al tratamiento con corticoides, en base a los criterios diagnósticos más recientes, es compatible con el de pancreatitis autoinmune y apoya la teoría de un proceso autoinmune sistémico. El leve aumento de la IgG indicaría un bajo grado de actividad, lo que concuerda con las escasas manifestaciones extrapancreáticas, apoyando por tanto las observaciones realizadas por otros autores. 
Aunque se ha sugerido que es una enfermedad progresiva recurrente, las series publicadas difieren en cuanto al porcentaje de recurrencias $(2,11)$ y los estudios de seguimiento a largo plazo son escasos (13). Un estudio demostró que la necesidad de tratamiento de mantenimiento era mayor en los pacientes con lesiones extrapancreáticas que en los que no las tenían (9). En nuestro paciente es llamativa la buena respuesta al tratamiento con corticoides y la resolución completa de todas las manifestaciones, que creemos es debida al inicio precoz del tratamiento cuando la enfermedad presentaba todavía una baja actividad. En el momento actual, tras 24 meses de seguimiento el paciente permanece asintomático, sin recurrencia de las lesiones pancreáticas ni extrapancreáticas, ni aparición de hallazgos típicos de pancreatitis crónica o insuficiencia pancreática.

En conclusión, la PAI es un tipo de pancreatitis crónica que posiblemente forme parte de una enfermedad sistémica autoinmune que puede afectar a otros órganos y tejidos, con mayor frecuencia la vía biliar extrapancreática y la aparición de fibrosis retroperitoneal. El inicio de tratamiento precoz con corticoides puede favorecer, como se demuestra en nuestro caso, la resolución completa de las manifestaciones, principalmente en los casos que en los que todavía no exista un grado alto de actividad.

\section{BIBLIOGRAFÍA}

1. Hamano H, Arakura N, Muraki T, Ozaki Y, Kiyosawa K, Kawa S. Prevalenceand distribution of extrapancreatic lesions complicating autoimune pancreatitis. J Gastroenterol 2006; 41: 1197-205.

2. Kawa S, Hamano H. Clinical features of autoinmune pancreatitis. J Gastroenterol 2007; 42 (Supl. 18): 9-14.

3. Sohn JH, Ho Byun J, Eon Yoon S, Choi EK, Park SH, Kim MH, et al. Abdominal extrapancreatic lessions associated with autoimmune pancreatitis: radiological findings and changes after therapy. Eur J Radiol 2007; doi:10.1016/j.ejrad.2007.08.018. (article in press).

4. Ohara H, Nakazawa T, Ando T, Joh T. Systemic extrapancreatic lesions associated with autoinmune oancreatits. J Gastroenterol 2007; 42 (Supl. 18): 15-21.

5. Sarles H, Sarles JC, Muratore R, et al. Chronic inflamatory sclerosis of the pancreas- an autonomous pancreatic disease? Am J Dig Dis 1961; 6: 688-98.

6. Kim MH, Kwon S. Diagnostic criteria for autoimmune chronic pancreatitis. J Gastroenterol 2007; 42 (Supl. 18): 42-9.

7. OKamisawa T, Matsukawa M, Ohkawa M. Autoimmune pancreatitis associated with retroperitoneal fibrosis. J Pancreas (On line) 2005; 6 (3): 260-3.

8. Kamisawa T, Yui Chen P, Tu Y, Nakajima H, Egawa N. Autimmune pancreatitis metachronously associated with retroperitoneal fibrosis with IgG4-positive plasma cell infiltration. World J Gastroenterol 2006; 12 (18): 2955-7.

9. Ohara H, Nakazawa T, Ando T, Joh T. Systemic extrapancreatic lesions associated with autoimmune pancreatitis. J Gastroenterol 2007; 42 (Supl. 18): 15-21.

10. Fukui T, Okazaki K, Yoshizawa H, Ohashi S, Tamaki H, Kawasaki $\mathrm{K}$, et al. A case of autoimmnune pancreatitis associated with sclerosing cholangitis, retroperitoneal fibrosis and Sjögren's syndrome. Pancreatology 2005; 5: 86-91.

11. Kuwatani M, Kawakami H, Makiyama H, Onodera M, Matsumoto K, Karasawa G, et al. Autoimmune pancreatitis with retroperitoneal fibrosis which responded to steroid therapy but was complicated with refractory renal dysfunction. Internal Med 2007; 46 (18): 1557-64.

12. Kamisawa T, Funata N, Hayashi Y, Eishi Y, Koike M, Tsuruta K, et al. A new clinicopathological entity of IgG4-related autoimmune disease. J Gastroenterol 2003; 38: 982-4.

13. Hirano K, Tada M, Isayama H, Yagioka H, Sasaki T, Kogure H, et al. Long-term prognosis of autoimmune pancreatitis with and without corticosterid treatment. Gut 2007; 56: 1719-24. 